\title{
Role of Brain-Derived Neurotrophic Factor in the Circadian Regulation of the Suprachiasmatic Pacemaker by Light
}

\author{
Fong-Qi Liang, Gregg Allen, and David Earnest \\ Texas A\&M University Health Science Center, Department of Human Anatomy and Medical Neurobiology, College of \\ Medicine, College Station, Texas 77843-1114
}

The central pacemaker located in the suprachiasmatic nucleus (SCN) of the hypothalamus mediates the generation of mammalian circadian rhythms, including an oscillation in pacemaker sensitivity to photic signals conveyed by the retinohypothalamic tract. Because brain-derived neurotrophic factor (BDNF) has been implicated in the functional regulation of neural input to other targets of visual pathways, the present study examined whether changes in BDNF expression or blockade of its action in the SCN affect circadian pacemaker responses to light. In rats receiving infusion of exogenous BDNF into the SCN, the free-running rhythm of activity in constant darkness was characterized by large phase advances in response to light exposure during the midsubjective day, when the circadian pacemaker is normally insensitive to photic perturbation. In contrast, SCN infusion of BDNF did not potentiate either phase-delaying or phase-advancing effects of light on the rat activity rhythm during the subjective night. In heterozygous BDNF mutant mice, deficits and damped rhythmicity in SCN levels of this neurotrophin were accompanied by marked decreases in the amplitude of light-induced phase shifts during the subjective night. In agreement with the effects of decreased BDNF expression, SCN infusion of the tyrosine kinase inhibitor K252a blocked or strongly inhibited both the phase-delaying and -advancing effects of light during the subjective night. Collectively, these findings suggest that BDNF-mediated signaling may play an important role in the circadian regulation of SCN pacemaker sensitivity to light.

Key words: brain-derived neurotrophic factor; neurotrophins; growth factors; tyrosine kinase receptors; circadian rhythms; suprachiasmatic nucleus; photoentrainment
In mammals, the suprachiasmatic nucleus (SCN) functions as an internal biological clock governing the generation and photic entrainment of circadian rhythms (Moore, 1983). Entraining light signals are transmitted to the SCN by the retinohypothalamic tract (RHT), a direct projection from a subset of retinal ganglion cells that mainly terminates within the ventrolateral SCN. Circadian photoentrainment occurs because the SCN pacemaker is reset by light during the subjective night (i.e., active phase of a nocturnal animal) but is insensitive to photic manipulation during the subjective day (Takahashi et al., 1984). Recent evidence of time-dependent changes in the effects of optic nerve stimulation on SCN neuronal activity suggests that this rhythm of pacemaker sensitivity to light may involve the differential regulation of RHT photic input and/or SCN cell responses to such information (Cui and Dyball, 1996).

Specific members of the neurotrophin family that includes nerve growth factor (NGF), brain-derived neurotrophic factor (BDNF), neurotrophin-3, and neurotrophin-4/5 have been implicated in the regulation of circadian pacemaker function by localization of these neurotrophins or their receptors in the SCN region (Sofroniew et al., 1989; Lehman et al., 1996; Liang et al., 1998 b). BDNF provided a central focus in the present analysis because involvement of this neurotrophin and its cognate receptor the TrkB tyrosine kinase in visual system function is suggested by studies demonstrating the following: the localization of

Received Nov. 30, 1999; revised Jan. 18, 2000; accepted Feb. 1, 2000.

We thank Rodney Walline for excellent technical assistance.

Correspondence should be addressed to Dr. David J. Earnest, Texas A\&M University Health Science Center, Department of Human Anatomy and Medical Neurobiology, 238 Reynolds Medical Building, College Station, TX 77843-1114. E-mail: dearnest@tamu.edu.

Copyright (C) 2000 Society for Neuroscience $0270-6474 / 00 / 202978-10 \$ 15.00 / 0$
TrkB receptors on retinal fibers near BDNF-expressing cells in their target areas (Jelsma et al., 1993; Pearson et al., 1995), structural and functional effects of BDNF on retinal ganglion cells and the visual cortex (Cohen-Cory and Fraser, 1995; Akaneya et al., 1997), and the photic induction of BDNF and trkB mRNA expression within the visual cortex (Castren et al., 1992). A role for BDNF in the photic regulation of the SCN is indirectly supported by our anatomical observation that BDNF-expressing cells in the ventrolateral SCN are closely apposed to TrkBimmunoreactive fibers emanating from the optic chiasm (Liang et al., 1998b). Coupled with increasing evidence of BDNF function in the short-term control of neuronal signaling within the brain (Kang and Schuman, 1995; Korte et al., 1995; Levine et al., 1995), these findings raise the possibility that BDNF may be involved in gating photic input to the SCN and its phase-shifting effects on circadian rhythms. Consistent with its potential function in regulating pacemaker responses to light, BDNF is rhythmically expressed within the SCN such that levels are elevated during the subjective night, when light shifts the phase of circadian rhythms, but remain low throughout the subjective day, when the clock mechanism is insensitive to photic perturbation (Liang et al., 1998a). If this relative amplification of BDNF-mediated signaling during the subjective night is an important process in the circadian sensitivity of the pacemaker mechanism to photic signals, then alterations in BDNF expression or inhibition of its action on TrkB receptors within the SCN should produce predictable changes in the resetting effects of light on circadian rhythms. To test this hypothesis, three experiments were conducted to determine (1) whether exogenous BDNF administration enables light to induce phase shifts during the subjective day and whether the resetting action of light during the subjective night is impaired in 
(2) heterozygous BDNF-deficient knock-out mice and (3) rats receiving the tyrosine kinase inhibitor K252a. BDNF and K252a were infused into the SCN because their short-lived biological activity in vivo has warranted this approach in comparable studies (Altar et al., 1992; Croll et al., 1994) and acute injections were ineffective in preliminary analyses.

\section{MATERIALS AND METHODS}

\section{Animals and housing conditions}

Adult male Sprague Dawley rats (175-200 gm) were purchased from Harlan Sprague Dawley (Indianapolis, IN). Adult male wild-type (C57/ B16) and heterozygous BDNF null mutant (Conover et al., 1995) mice were obtained from Regeneron Pharmaceuticals (Tarrytown, NY). All animals were housed individually in cages equipped with running wheels so that the circadian rhythm of wheel-running activity could be continuously recorded. Before experimentation, animals were maintained under a standard $12 \mathrm{hr}$ photoperiod [light/dark cycle of 12:12 hr (LD 12:12); lights on at 06:00 hr], and baseline activity behavior was recorded for 10-14 d. Periodic animal care and experimental manipulations in constant darkness (DD) were accomplished using an infrared viewer (FJW Optical Systems). Animals were provided food and water ad libitum.

\section{SCN cannulation for chronic infusion or acute injection}

Sprague Dawley rats were anesthetized (ketamine, $87 \mathrm{mg} / \mathrm{kg}$, and xylazine, $1.3 \mathrm{mg} / \mathrm{kg}$ ), and a stainless steel cannula with an osmotic pump connector (28 gauge; Plastic One, Roanoke, VA) was stereotaxically implanted near the ventral extent of the third ventricle to allow drug infusion into the SCN. Empirical stereotaxic coordinates for the SCN region $(1.3 \mathrm{~mm}$ posterior to bregma and $7.5 \mathrm{~mm}$ ventral to the dura along the midline) were derived from those established by Paxinos and Watson (1997).

Three days after cannula placement surgery, a miniosmotic pump (model 2004; Alzet) was implanted subcutaneously in the intrascapular region of anesthetized rats (ketamine, $87 \mathrm{mg} / \mathrm{kg}$, and xylazine, 1.3 $\mathrm{mg} / \mathrm{kg}$ ) and attached to the connector cannula via polyethylene 60 (PE60) tubing. Osmotic pumps were filled with recombinant human BDNF $(10 \mu \mathrm{g})$ or K252a (467 ng) diluted in $200 \mu \mathrm{l}$ of artificial CSF $(\mathrm{aCSF}), \mathrm{pH}=7.4$. On the basis of the operational specifications of this osmotic pump (delivery rate $=0.25 \mu \mathrm{l} / \mathrm{hr}$ ), $250 \mathrm{ng}$ of BDNF or $56.04 \mathrm{ng}$ of K252a was infused into the SCN region per day for a maximum duration of $28 \mathrm{~d}$. Control animals received osmotic pump infusion of aCSF alone into the $\mathrm{SCN}$ region.

For acute injection of $\mathrm{BDNF}$ or $\mathrm{K} 252 \mathrm{a}$ into the $\mathrm{SCN}$, rats were implanted with a stainless steel guide cannula (24 gauge; Plastic One). Stereotaxic coordinates were similar to those for placement of the osmotic pump cannula, except that the guide cannula was implanted (6.5 $\mathrm{mm}$ ventral to the dura) to allow extension of an internal injector stylus (33 gauge) into the parenchyma (by $1 \mathrm{~mm}$ ). A compatible dummy stylet was inserted into each guide cannula to prevent debris entry.

\section{Histological analysis}

At the conclusion of behavioral analysis, anesthetized animals (sodium pentobarbital, $3.0 \mathrm{mg} / \mathrm{kg}$ ) were injected via the guide cannula with fluorogold (2\%; Fluorochrome, Englewood, CA) and killed by transcardiac perfusion with $50 \mathrm{ml}$ of $0.1 \mathrm{M}$ phosphate buffer, $\mathrm{pH}=7.3$, containing heparin, followed by $200-250 \mathrm{ml}$ of $4 \%$ paraformaldehyde. Immediately after perfusion, the brains were removed, post-fixed for $1-2 \mathrm{hr}$ at $4^{\circ} \mathrm{C}$, and stored overnight in cryoprotectant solution $(15 \%$ sucrose in $0.15 \mathrm{M}$ phosphate buffer). The tissue was then frozen and sectioned in the coronal plane at $30 \mu \mathrm{m}$ using a sliding microtome. Cannula placement was determined by fluorogold localization in mounted sections using fluorescence microscopy. Only data from animals with verified cannula placement in the SCN region were used for analysis of treatment effects on circadian wheel-running behavior.

\section{Experimental protocols}

After completion of all surgical procedures, animals were exposed to DD. Animals were allowed to free-run in DD for at least 2 weeks before experimental manipulations. Light pulses (150-200 lux) were delivered by transferring the home cages of individual animals to a ventilated, light-proof chamber. After experimental treatment, animals were allowed to free-run in DD without any further perturbation.

Experiment 1: effect of BDNF infusion into the SCN on light-induced phase shifts of the rat activity rhythm. Effects of BDNF on the resetting of the SCN pacemaker by light at different circadian times (CT) were examined in male rats receiving an osmotic pump infusion of either aCSF or BDNF $(250 \mathrm{ng} / \mathrm{d})$. Control $(n=10)$ and BDNF-treated $(n=$ 13) animals were subjected to a 60 min light pulse $\sim 6 \mathrm{hr}$ before the onset of activity (CT 6) because light exposure at this time is known to have no phase-shifting effect on rodent circadian rhythms (Summer et al., 1984; Takahashi et al., 1984; Schwartz and Zimmerman, 1990). Exclusion of two aCSF-infused and four BDNF-treated rats from analysis was necessary because treatment was disrupted by cannula failure or because arrhythmicity or irregular activity onsets compromised the evaluation of phase shifts in the activity pattern. To determine whether BDNF can potentiate light-induced phase shifts during the subjective night, additional groups of animals receiving SCN infusion of $\operatorname{BDNF}(n=6)$ or aCSF $(n=5)$ were exposed to a 20 min light pulse at either 2 or $10 \mathrm{hr}$ after the onset of activity (CT 14 or 22) because large phase delays or advances of the rat activity rhythm occur after light treatment at these circadian times (Summer et al., 1984). Exclusion of one BDNF-treated rat from analysis was necessary because arrhythmicity compromised the evaluation of light-induced phase shifts in the activity pattern at CT 22 . All experimental and control animals received light treatment only once during the 4 week period of infusion.

In parallel with this behavioral analysis, SCN content of BDNF was examined by ELISA in auxiliary groups of control and experimental rats to determine the effects of the infusion paradigm on BDNF levels and their rhythmic pattern in the SCN. Male rats receiving an infusion of either aCSF $(n=28)$ or BDNF $(250 \mathrm{ng} / \mathrm{d} ; n=28)$ for $14 \mathrm{~d}$ (i.e., midpoint of minipump functional duration) under LD 12:12 conditions were exposed to DD (beginning at CT 12) and $12 \mathrm{hr}$ later killed at $4 \mathrm{hr}$ intervals for $24 \mathrm{hr}$ by decapitation using an infrared viewer. SCN tissue was immediately dissected as described previously (Earnest and Sladek, 1986) and stored at $-80^{\circ} \mathrm{C}$ until subsequent assay of BDNF content.

To determine whether BDNF alone has any phase-shifting effect on the circadian rhythm of wheel-running activity, acute injections $(1 \mu \mathrm{l})$ of BDNF (40 ng) or aCSF were administered at either CT 6, 14, or 22 into the SCN region of rats maintained in $\mathrm{DD}(n=18)$. These injections were delivered under light anesthesia (Metofane; Mallinckrodt Veterinary) via the guide cannula using a $10 \mu$ l Hamilton syringe with connector tubing (PE80) and an internal injector stylus. The injection volume was delivered slowly (1-2 min interval), and then the internal cannula was left in place for an equivalent period of time to allow drug diffusion. The animals were then allowed to free-run in DD for at least $17 \mathrm{~d}$ before receiving a second treatment. Experiments used a crossover design such that each animal received only two injections, one with BDNF and another with aCSF.

Experiment 2: effect of decreased BDNF expression on light-induced phase shifts of the activity rhythm in heterozygous mutant mice. To determine whether decreased BDNF expression within the SCN will abate the phase-shifting effect of light, mice heterozygous for the BDNF null mutation $\left(b d n f^{+/-}\right)$were examined for their circadian responses to light during the subjective night. In $b d n f^{+/-}$mice, both the levels of BDNF protein and neuronal number in different sensory ganglia are decreased by $\sim 50 \%$, but body mass, structural organization of the CNS, and motor neuron development or function show no gross abnormalities (Jones et al., 1994; Conover et al., 1995; Korte et al., 1995; Bianchi et al., 1996). After recording baseline activity in LD 12:12 (lights on at 06:00 hr), wild-type mice $(n=28)$ and $b d n f^{+/-}(n=28)$ were maintained in DD. After establishment of a stable free-running period in DD $(\approx 2-3$ weeks $)$, animals were subjected to 20 min light pulses at either CT 6,14 , or 22 because light exposure at these times induces no shift, delays, or advances in the phase of the mouse activity rhythm (Schwartz and Zimmerman, 1990). All wild-type and mutant mice were only subjected to light treatment on two different occasions during the course of this experiment. At the conclusion of behavioral analysis, animals were first housed under LD 12:12 conditions for $14 \mathrm{~d}$ to provide for stable entrainment of the activity rhythm and then exposed to DD (at 18:00 hr). Twelve hours later, groups of wild-type and mutant mice (each $n=4)$ were killed at 4 $\mathrm{hr}$ intervals for $24 \mathrm{hr}$ by decapitation using an infrared viewer. The SCN was immediately dissected, frozen in liquid nitrogen, and later extracted to recover total RNA and soluble proteins. BDNF content in recovered protein samples was determined using a sandwich ELISA protocol ( $\mathrm{Li}-$ ang et al., 1998a,b).

Experiment 3: effect of $\mathrm{K} 252 \mathrm{a}$ on light-induced phase shifts of the rat activity rhythm. Complementary to the analysis of decreased BDNF expression in mutant mice, studies were performed to determine whether 
the phase-shifting effects of light on the rat activity rhythm are impaired by SCN infusion of K252a, an alkaloid compound that inhibits the phosphorylation of all tyrosine kinase receptors (Berg et al., 1992; Tapley et al., 1992). Use of K252a in the present context is appropriate because this tyrosine kinase receptor inhibitor has been shown to block BDNFinduced enhancement of neuronal signaling within the brain (Kang and Schuman, 1995; Levine et al., 1995; Akaneya et al., 1997). Adult male rats were implanted with an indwelling cannula to provide for the chronic inf usion of aCSF $(n=12)$ or K252a $(56.04 \mathrm{ng} / \mathrm{d} ; n=12)$ via an osmotic minipump for $28 \mathrm{~d}$. After establishment of a stable free-running period in DD $(\approx 2-3$ weeks), animals were exposed to 30 min light pulses at either CT 14 or 22 because photic stimulation at these circadian times is known to induce maximal phase delays or advances (Summer et al., 1984), respectively. All animals received light treatment only once during the experiment. Exclusion of three aCSF-infused rats and one K252atreated animal from analysis was necessary because treatment was disrupted by cannula failure or arrhythmicity or irregular activity onsets compromised the evaluation of phase shifts in the activity pattern.

To determine whether K252a alone has any phase-shifting effect on the circadian rhythm of wheel-running activity, acute injections $(1 \mu \mathrm{l})$ of K252a (9.34 ng) or aCSF were administered at either CT 14 or 22 into the $\mathrm{SCN}$ region of rats maintained in DD $(n=12)$. K252a was injected using procedures described in Experiment 1 for BDNF injections.

\section{Analysis of circadian behavior}

Wheel-running activity was continuously recorded, summed, and stored in 10 min bins using a computer running Dataquest IV data acquisition software (Data Sciences). Graphical records of circadian activity rhythms were generated and analyzed using Tau software. Light- and druginduced shifts in the circadian phase of free-running activity rhythms in DD were assessed as described previously (Ellis et al., 1982) using guide lines through steady-state activity onsets during $10-15 \mathrm{~d}$ of the pretreatment and post-treatment intervals for reference. The phase-shift amplitude was derived from the difference between the expected time of activity onset as projected using pretreatment reference points and the actual time of activity onset after post-treatment restoration of steadystate circadian period $(\tau)$. Shifts in the circadian phase of the activity rhythm were independently assessed in this manner by two experienced individuals, and reported values reflect the averages of their determinations. By the use of the chi-square periodogram (Tau) and fast Fourier transform analyses, steady-state $\tau$ of the activity rhythm in DD was also determined in all animals to provide several comparisons: before and after light treatment within individual animals and between control and experimental groups during infusion treatment. Animals showing highly irregular onsets of activity, arrhythmicity, or cannula failure were excluded from analysis. Statistical analyses were performed on the raw data using a pooled $t$ test to determine the significance of treatment effects on phase shifts induced by light and $\tau$ of the activity rhythm (see Figs. $1 B, 2,4 B, 6 B$ ).

\section{$B D N F$ and $K 252 a$}

Recombinant human (rh) BDNF (PeproTech) was diluted to $41.7 \mathrm{ng} / \mu \mathrm{l}$ in aCSF containing $0.1 \%$ BSA before loading into osmotic minipumps (Alzet). Previous reports on the stability of BDNF during osmotic pump delivery at $37^{\circ} \mathrm{C}$ indicate that this neurotrophin retains at least $80 \%$ of its bioassayable activity in comparison with stock solutions that had been stored at $4^{\circ} \mathrm{C}$ (Altar et al., 1992). The tyrosine kinase inhibitor K252a (Calbiochem, La Jolla, CA) was diluted to $9.34 \mathrm{ng} / \mu \mathrm{l}(20 \mu \mathrm{M})$ in dimethylsulfoxide (DMSO; Sigma, St. Louis, MO) and then loaded into minipumps.

\section{BDNF ELISA}

For analysis of BDNF content by ELISA, SCN tissue from individual animals was homogenized in TRIzol reagent (Life Technologies, Gaithersburg, MD). Soluble proteins were recovered from the organic phase by sequential precipitation (Chomczynski, 1993) and dissolved in 1\% SDS. Before ELISA analysis, the protein content in each sample was determined by the bicinchoninic acid method (Micro BCA; Pierce, Rockford, IL).

BDNF content in recovered protein samples was determined using a sandwich ELISA protocol. Ninety-six-well microplates (Nunc, Naperville, IL) were coated overnight at $4^{\circ} \mathrm{C}$ with $100 \mu \mathrm{l}$ of rabbit anti-BDNF antibody (Santa Cruz Biotechnology, Santa Cruz, CA) diluted (1:250) in $0.025 \mathrm{M}$ carbonate-bicarbonate buffer, $\mathrm{pH}$ 9.7. With interceding washes [50 mm Tris-buffered saline (TBS), $\mathrm{pH} 7.4$ ], the plates were subjected to sequential $2 \mathrm{hr}$ incubations at room temperature with blocking solution ( $1 \%$ bovine serum albumin in TBS), triplicate aliquots of tissue protein samples or BDNF standards (0.1-100 ng/well), chicken anti-BDNF antibody (1:500; Promega, Madison, WI), and alkaline phosphataseconjugated goat anti-chicken IgY (1:1000; Promega). Alkaline phosphatase activity was detected using $p$-nitrophenyl phosphate (Pierce) dissolved in $0.1 \mathrm{~m}$ bicarbonate buffer, $\mathrm{pH} 10$, as the color substrate. After color development (40-50 min), the absorbance at $405 \mathrm{~nm}$ was measured using a plate reader (Bio-Tech). By the use of serial dilutions of known amounts of rhBDNF, this color reaction yielded a linear standard curve from 0.5 to $100 \mathrm{ng} / \mathrm{ml}$. The intra-assay and interassay coefficients of variation were $<5$ and $10 \%$, respectively. BDNF content in SCN lysates was quantified within the linear range of a standard curve and normalized for the soluble protein (micrograms) that was assayed in each sample. The rabbit and chicken anti-BDNF antibodies used in this assay show $<2 \%$ cross-reactivity with NGF, neurotrophin-3, or neurotrophin$4 / 5$ (at concentrations as high as $10 \mu \mathrm{g} / \mathrm{ml}$ ). In addition, the specificity of these antibodies for BDNF has been verified previously by Western blot analysis (Liang et al., 1998b).

Within a treatment group, the mean values for time-point determinations of BDNF protein levels in the SCN were evaluated for evidence of temporal variation using one-way ANOVA. Paired comparisons of BDNF levels at specific circadian times were analyzed post hoc for statistical differences using the Newman-Keuls sequential range test. In addition, statistical analysis was performed using a pooled $t$ test to determine whether BDNF levels at each time point were significantly different between control and experimental groups (see Figs. 3, 5).

\section{RESULTS}

\section{Experiment 1: effect of BDNF infusion into the SCN on light-induced phase shifts of the rat activity rhythm}

During the $28 \mathrm{~d}$ infusion interval, both aCSF- $(n=8)$ and BDNF-treated $(n=9)$ rats showed clear free-running activity rhythms in DD with similar periodicities (mean $\tau=24.00 \pm 0.04$ vs $24.06 \pm 0.07 \mathrm{hr}$, respectively). Although BDNF had no significant effect on the steady-state circadian period $(p>0.05)$, SCN infusion of this neurotrophin did alter the effect of light on the activity rhythm during the midsubjective day. Exposure to a $1 \mathrm{hr}$ light pulse at CT 6 induced large phase advances (mean $\Delta \Phi=$ $+2.28 \pm 0.35 \mathrm{hr}$ ) of the activity rhythm in BDNF-infused rats but had little or no phase-shifting effect (mean $\Delta \Phi=+0.25 \pm 0.12$ hr) on the circadian behavior of animals receiving aCSF (Fig. 1). Light-induced phase shifts at CT 6 in BDNF-treated rats were significantly greater $(p<0.01)$ than those observed in control animals. These advancing shifts in BDNF-treated animals were often distinguished by a large number of transient cycles (7-20) before a new steady state was reached. At the conclusion of these transients, the steady-state period of the activity rhythm in BDNF-infused rats was similar to that expressed before light exposure (mean $\tau=24.06 \pm 0.07 \mathrm{vs} 24.05 \pm 0.09 \mathrm{hr}$, respectively), indicating that this effect truly reflects a phase shift, not a longterm change in circadian period, in response to light. Importantly, acute injection of BDNF alone (40 ng in $1 \mu \mathrm{l})$ into the SCN at CT 6 without exposure to light had no significant effect $(p>0.05)$ on the circadian phase of the rat activity rhythm (mean $\Delta \Phi=$ $+0.33 \pm 0.20 \mathrm{hr}$ ) relative to that of matched aCSF controls (mean $\Delta \Phi=+0.23 \pm 0.10 \mathrm{hr})$.

In contrast to its modulatory effects on circadian responses to light at CT 6, BDNF infusion did not alter light-induced phase shifts of the rat activity rhythm at CT 14 or 22 . In both BDNF$(n=11)$ and aCSF-treated $(n=10)$ animals, a 20 min light exposure induced phase delays at CT 14 (Fig. 2; mean $\Delta \Phi=$ $-2.42 \pm 0.26 \mathrm{vs}-2.65 \pm 0.27 \mathrm{hr}$, respectively) and advances at CT 22 of comparable amplitude (mean $\Delta \Phi=+2.14 \pm 0.2$ vs $+2.26 \pm 0.46 \mathrm{hr}$, respectively). No significant differences in the phase-shifting responses to light at either time $(p>0.05)$ were 


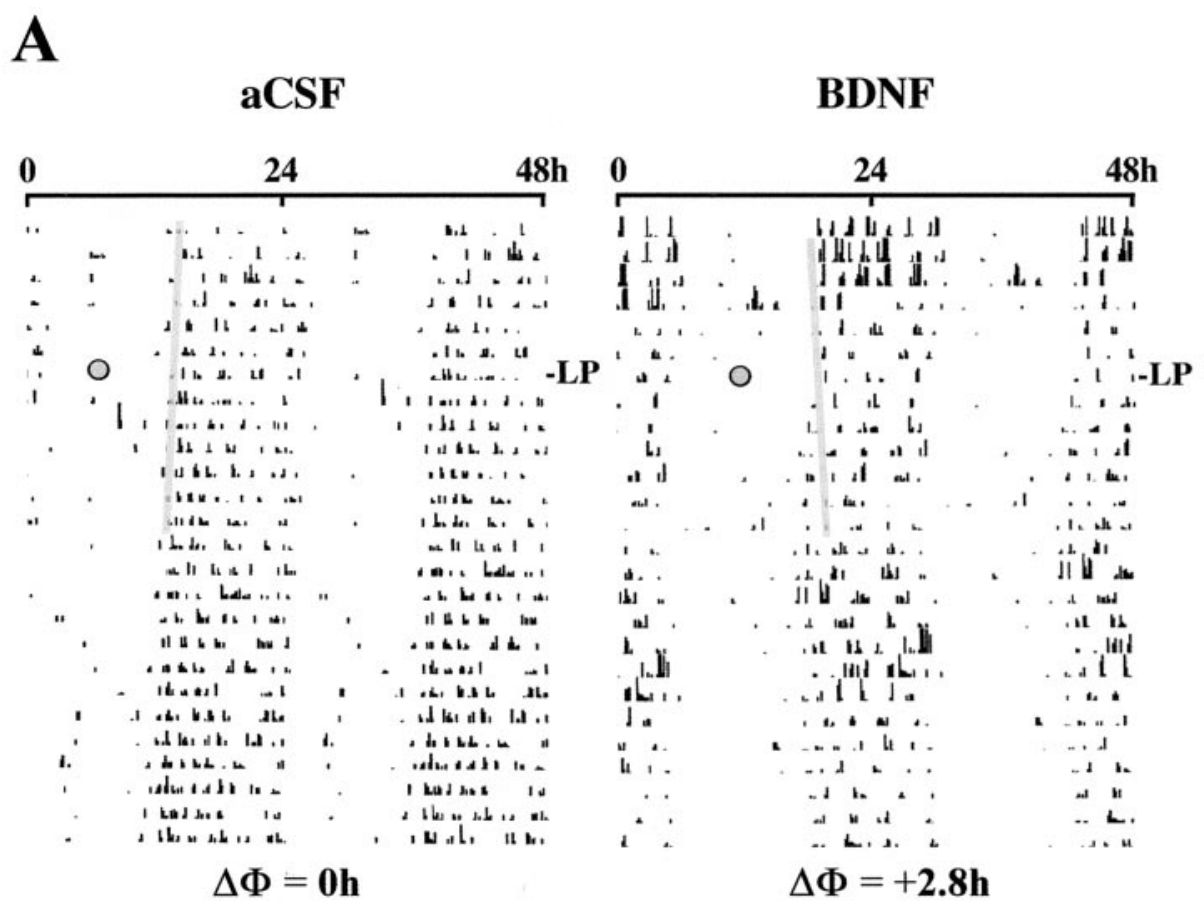

B

0

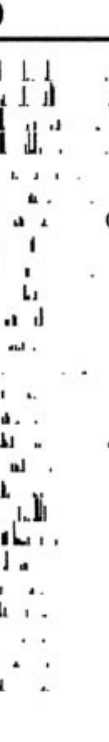

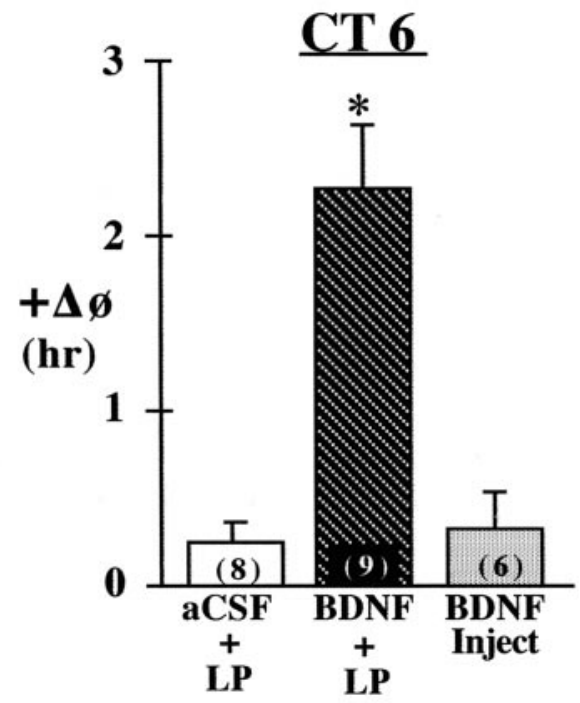

Figure 1. Effects of BDNF infusion on the responses of the rat activity rhythm to light at CT 6 . $A$, Representative activity records of two adult male rats receiving infusion of either aCSF or BDNF into the SCN during exposure to DD. Actograms are double-plotted over a 48 hr period, and shaded circles on each record indicate the day and time (CT 6) during which animals were exposed to a $1 \mathrm{hr}$ light pulse $(L P)$. A thin shaded bar has been fitted through the activity onsets for 5-6 d before the light pulse to facilitate visualization of phase shifts $(\Delta \Phi)$ in the activity rhythm. $B$, Mean $( \pm$ SEM) phase advance $(+\Delta \Phi)$ in hours of the activity rhythm induced by a $1 \mathrm{hr}$ light pulse at CT 6 in rats receiving SCN infusion of aCSF $(a C S F+L P)$ or BDNF $(B D N F+L P)$ or by a single injection of BDNF $(40 \mathrm{ng})$ alone at CT 6 without exposure to light (BDNF Inject). For each treatment group, the sample size is designated in parentheses on the bar. Light-induced phase shifts were significantly greater $\left({ }^{*} p<0.01\right)$ in BDNF-infused rats relative to those in animals receiving aCSF infusion or BDNF injection alone.

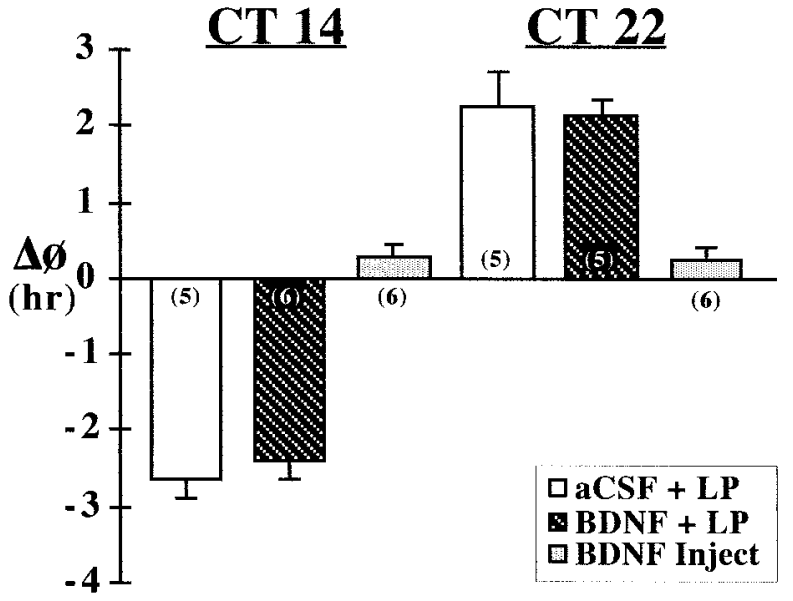

Figure 2. Effects of BDNF infusion on the responses of the rat activity rhythm to light at CT 14 and 22. Mean $( \pm$ SEM) phase shift $(\Delta \Phi)$ in hours of the activity rhythm induced by a 20 min light pulse $(L P)$ at CT 14 or 22 in rats receiving $\mathrm{SCN}$ infusion of aCSF $(a C S F+L P)$ or $\mathrm{BDNF}$ $(B D N F+L P)$ or by a single injection of BDNF (40 ng) alone at CT 14 or 22 without exposure to light (BDNF Inject). For each treatment group, the sample size is designated in parentheses. Phase delays are indicated by negative values, and advances are denoted by positive values.

detected between BDNF- and aCSF-infused rats. For both experimental and control groups, phase delays of the activity rhythm were complete within one cycle, whereas advancing shifts required four to six transient cycles before steady-state $\tau$ was re-established.
Effects of the infusion paradigm on BDNF levels and their rhythmic pattern in the SCN were analyzed in parallel groups of animals. Control rats receiving infusion of aCSF exhibited a rhythmic pattern of BDNF content in the SCN similar to that described previously for normal intact rats (Liang et al., 1998a). SCN levels of BDNF protein reached peak values during the subjective night and were low throughout the subjective day (Fig. 3). In contrast, BDNF-infused rats showed no evidence of rhythmic fluctuation in SCN content of BDNF. Instead, BDNF levels in the SCN of these animals constantly remained at higher levels than those observed in aCSF-treated control rats. $\mathrm{BDNF}$ levels in the $\mathrm{SCN}$ at times throughout the subjective day (CT 0,4 , and 8$)$ were significantly greater $(p<0.05)$ in BDNFinfused rats than in control animals. During the subjective day, experimental and control animals were distinguished by a fourfold to fivefold difference in SCN content of BDNF, primarily because of the rhythmic decline occurring only in aCSF-treated animals.

\section{Experiment 2: effect of decreased BDNF expression on light-induced phase shifts of the activity rhythm in heterozygous mutant mice}

The absolute levels and rhythmic patterns of wheel-running activity (Fig. 4) were comparable in wild-type $\left(b d n f^{+/+}\right)$and heterozygous BDNF knock-out $\left(b d n f^{+/-}\right)$mice. Under LD 12:12 conditions, $b d n f^{+/-}$mice and their wild-type counterparts showed similar patterns of entrainment such that the daily onset of activity occurred shortly after lights off (data not shown). In $\mathrm{DD}$, the free-running period of the activity rhythm in the $b d n f^{+/-}$ mice was significantly shorter $(p<0.01)$ than that observed in 


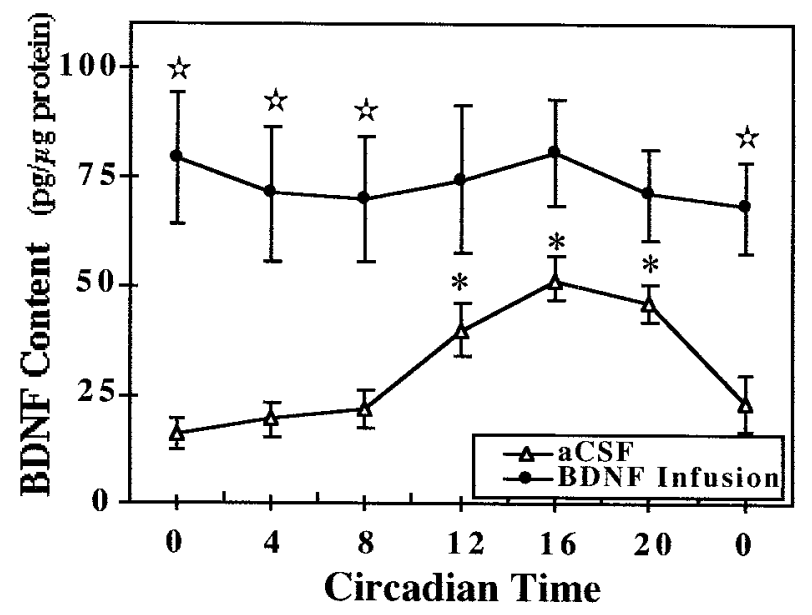

Figure 3. Temporal pattern of BDNF content in the SCN of rats receiving infusion of aCSF or BDNF. Symbols represent the mean values $( \pm \mathrm{SEM})$ of BDNF protein expression in the SCN of rats that were infused with aCSF or BDNF (250 ng/d) for $14 \mathrm{~d}$ and then killed at $4 \mathrm{hr}$ intervals $(n=4)$ in DD. At CT 12, 16, and 20, BDNF levels in aCSFinfused rats were significantly greater $\left({ }^{*} p<0.05\right)$ than those observed at all other times. Significant differences in the SCN content of BDNF $(\approx p<$ $0.05)$ were observed between aCSF- and BDNF-infused rats at CT 0,4 , and 8 .

wild-type mice (mean $\tau=23.23 \pm 0.08$ and $23.69 \pm 0.10 \mathrm{hr}$, respectively). Mutant $b d n f^{+/-}$mice were also distinguished by abated phase-shifting responses to light. As expected, 20 min light pulses induced clear phase delays at CT 14 (mean $\Delta \Phi=-2.66 \pm$ $0.57 \mathrm{hr}$ ) and large advances at CT 22 (mean $\Delta \Phi=+4.76 \pm 0.30$ hr) of the activity rhythm in all wild-type animals. In $b d n f^{+/-}$ mice, the same light stimulus produced only small phase delays at CT 14 (mean $\Delta \Phi=-1.26 \pm 0.13 \mathrm{hr}$ ) and had little or no phase-shifting effect at CT 22 (mean $\Delta \Phi=+0.53 \pm 0.35 \mathrm{hr}$ ). The mean phase delay at CT 14 and advance at CT 22 in $b d n f^{+/-}$mice were significantly decreased ( $p<0.05$ and $p<0.01$, respectively) relative to the light-induced shifts observed in wild-type mice at these circadian times (Fig. 4). Light exposure at CT 6 had a negligible effect on the phase of the activity rhythm in both wild-type and $b d n f^{+/-}$mice (mean $\Delta \Phi=+0.20 \pm 0.13$ and $+0.17 \pm 0.11 \mathrm{hr}$, respectively).

Wild-type and $b d n f^{+/-}$mice were also marked by differences in the relative levels and the temporal profile of BDNF content in the SCN. In wild-type mice, the SCN content of BDNF oscillated in a rhythmic manner similar to that reported previously in rats (see Liang et al., 1998a), with peak values during the subjective night (Fig. 5). One-way ANOVA indicated that determinations of $\mathrm{BDNF}$ protein levels in the SCN at $4 \mathrm{hr}$ intervals throughout the circadian cycle were significantly different $(F=15.81 ; p<0.01)$. Mean levels of BDNF protein in the SCN during the subjective night at CT 16 and 20 were significantly greater $(p<0.05)$ than those observed at all times throughout the subjective day (CT 0 , 4, and 8). The wild-type rhythm in the SCN expression of BDNF protein was characterized by a twofold difference between peak and minimum values. In $b d n f^{+/-}$mice, the temporal pattern of BDNF expression in the SCN followed a similar trend in which levels were higher during the subjective night than during the subjective day. However, no significant variation was observed between time-based determinations of BDNF levels in the SCN of mutant mice $(F=1.15 ; p>0.05)$. At all circadian times, the SCN content of BDNF in $b d n f^{+/-}$mice was significantly less $(p<0.05)$ than that found in wild-type mice, although this phenotypic difference was more pronounced during the subjective night. In accord with previous comparisons for sensory neurons (Bianchi et al., 1996), the SCN of mutant mice showed an overall decrease in BDNF protein expression of $\sim 50 \%$ in relation to that found in wild-type animals.

\section{Experiment 3: effect of K252a on light-induced phase shifts of the rat activity rhythm}

Throughout the $28 \mathrm{~d}$ treatment interval, rats receiving SCN infusion of K252a $(n=11)$ or aCSF $(n=9)$ showed clear free-running activity rhythms in DD with similar periodicities (mean $\tau=24.05 \pm 0.07$ and $24.03 \pm 0.06 \mathrm{hr}$, respectively). Although K252a infusion had no significant effect on the steadystate circadian period $(p>0.05)$, treatment with this tyrosine kinase inhibitor blocked or abated the phase-shifting effect of light on the activity rhythm during the subjective night (Fig. 6). In control animals receiving infusion of aCSF, light exposure induced large phase delays at CT 14 (mean $\Delta \Phi=-2.84 \pm 0.23 \mathrm{hr}$ ) and advances at CT 22 (mean $\Delta \Phi=+2.68 \pm 0.46 \mathrm{hr}$ ) in the circadian rhythm of activity. In contrast, 6 out of $11 \mathrm{~K} 252 \mathrm{a}-$ infused rats showed no phase shifts of the activity rhythm in response to light stimulation at CT 14 or 22. The average phase delays at CT 14 (mean $\Delta \Phi=-0.67 \pm 0.25 \mathrm{hr}$ ) and advances at CT 22 (mean $\Delta \Phi=-0.66 \pm 0.17 \mathrm{hr}$ ) in K252a-inf used rats were significantly less $(p<0.01)$ than the light-induced shifts observed in aCSF-inf used animals at these circadian times. Acute injection of K252a alone $(9.34 \mathrm{ng}$ in $1 \mu \mathrm{l}$ ) into the SCN at CT 14 or 22 without exposure to light had little effect on the circadian phase of the rat activity rhythm (mean $\Delta \Phi=-0.37 \pm 0.18$ and $+0.35 \pm$ $0.11 \mathrm{hr}$, respectively).

\section{DISCUSSION}

The present study provides multiple lines of evidence demonstrating that changes in BDNF expression or inhibition of its cognate receptor TrkB within the SCN alter the phase-shifting effects of light on circadian rhythms. First, administration of exogenous BDNF into the rat SCN transformed normal pacemaker responses to light, enabling the photic induction of large phase advances in the activity rhythm during the midsubjective day. Second, mutant $b d n f^{+/-}$mice exhibited deficits and damped rhythmicity in SCN expression of BDNF that were accompanied by corresponding decreases in the amplitude of light-induced phase shifts during the subjective night. In agreement with this observation, SCN infusion of the tyrosine kinase inhibitor K252a blocked or abated both phase delays and advances of the rat activity rhythm in response to light during the subjective night. In conjunction with previous evidence of the close association of BDNF-expressing cells in the ventral SCN with TrkBimmunoreactive fibers emanating from the optic chiasm and of the circadian expression of BDNF within the SCN (Liang et al., 1998a,b), these findings suggest that BDNF may play an important role in the time-dependent regulation of circadian rhythms by light.

Although "nonphotic" stimuli such as dark pulses, induced activity, social cues, and neuropeptide Y are known to induce phase advances of rodent rhythms directly during the subjective day (Boulos and Rusak, 1982; Albers and Ferris, 1984; Mrosovsky, 1988; Reebs and Mrosovsky, 1989), BDNF infusion in the present study is distinguished by its effect in engendering large phase shifts in response to light during this portion of the circadian cycle. Importantly, acute treatment with BDNF alone during 


\section{A}
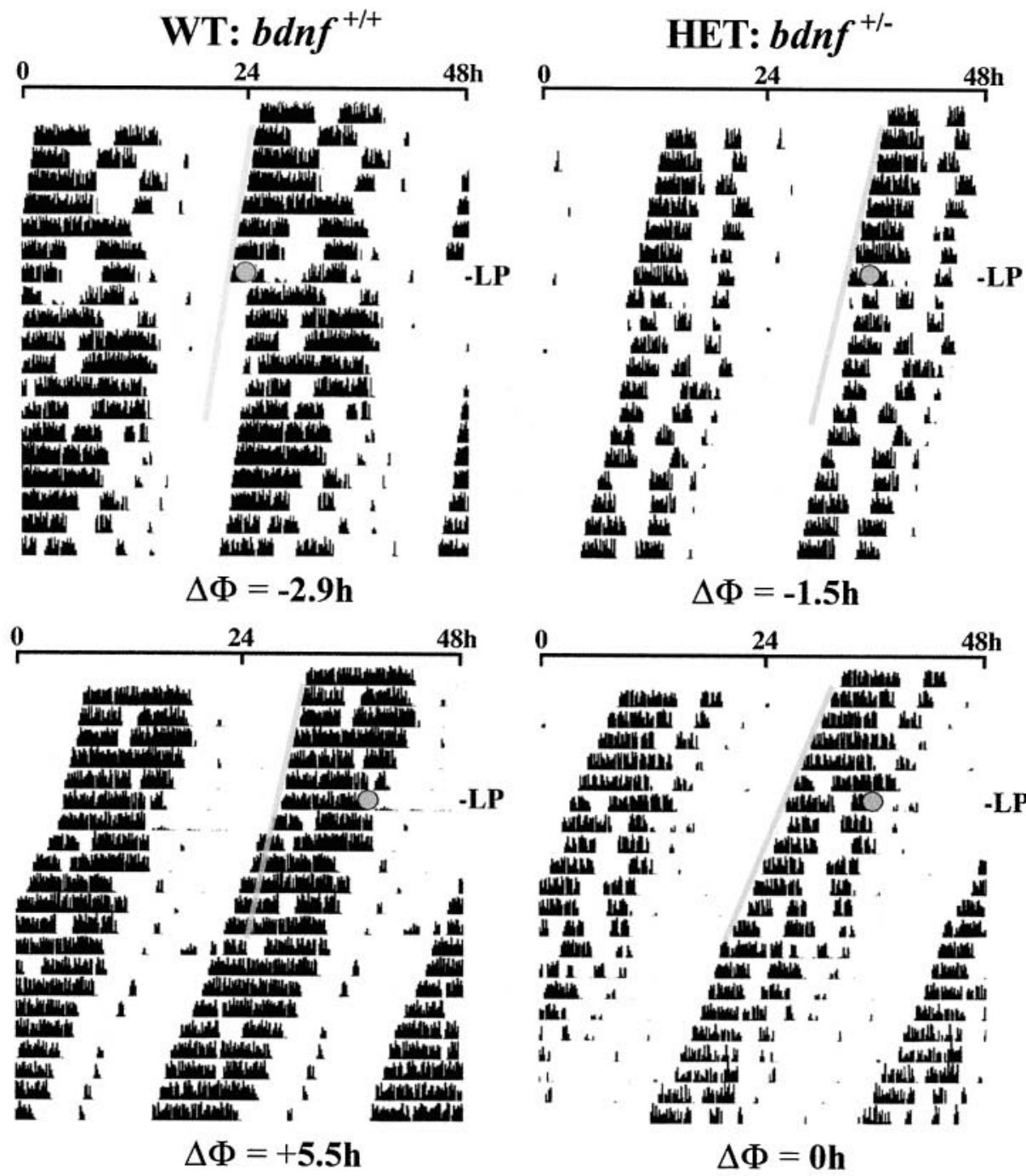

B

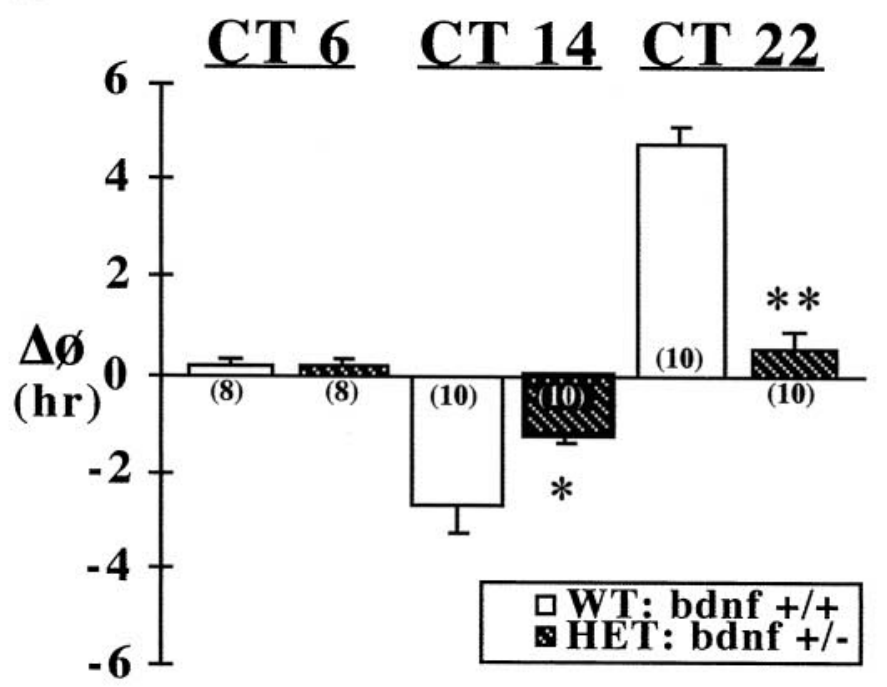

Figure 4. Effects of decreased BDNF expression in the mouse SCN on light-induced phase shifts $(\Delta \Phi)$ of the rhythm in wheel-running activity. $A$, Representative activity records in DD of wild-type mice ( $W T: b d n f^{+/+}$) and mice heterozygous for the BDNF null mutation (HET: $b d n f^{+/-}$) exposed to a $20 \mathrm{~min}$ light pulse $(L P)$ at CT 14 (top) or 22 (bottom). Actograms are double-plotted over a $48 \mathrm{hr}$ period, and shaded circles on each record indicate the day and time during which light pulses were administered. A thin shaded bar has been fitted through the activity onsets for $6 \mathrm{~d}$ before each light pulse to facilitate visualization of the phase shifts in the activity rhythm. $B$, Mean $( \pm$ SEM) phase shift $(\Delta \Phi)$ in hours of the activity rhythm induced by light pulses at CT 6, 14, and 22 in wild-type and $b d n f^{+/-}$ mice. For each treatment group, the sample size is designated in parentheses. Phase delays are indicated by negative values, and advances are denoted by positive values. Light-induced phase delays at CT $14(* p<0.05)$ and advances at CT $22(* * p<0.01)$ were significantly decreased in mutant $b d n f^{+1-}$ mice compared with those in wild-type mice. 


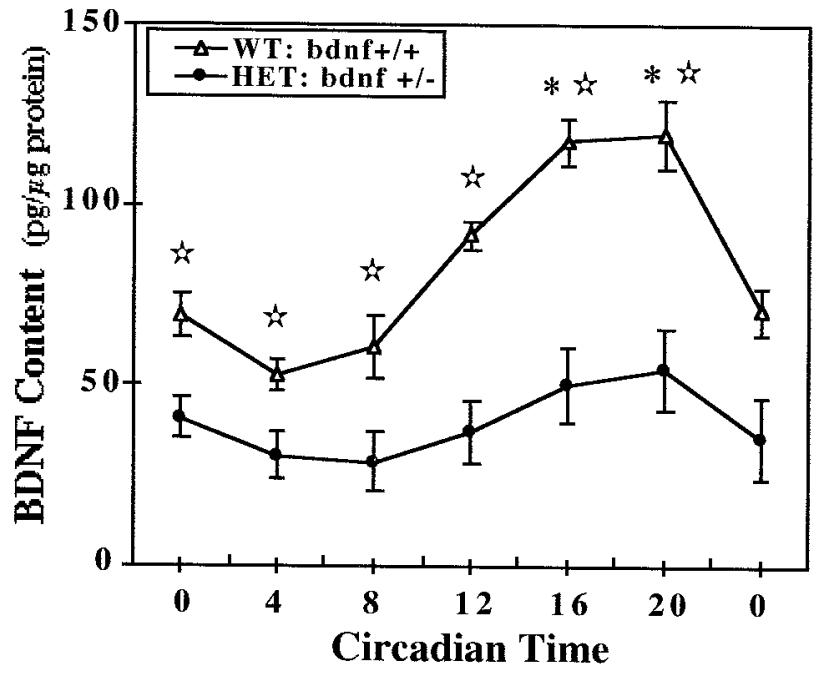

Figure 5. Temporal patterns of BDNF content in the SCN of wild-type and heterozygous BDNF knock-out mice. Symbols represent the mean values $( \pm$ SEM) of BDNF protein expression in the SCN of wild-type mice $\left(W T: b d n f^{+/+}\right)$and BDNF knock-out mice $\left(H E T: b d n f^{+-}\right)$killed at $4 \mathrm{hr}$ intervals $(n=4)$ in DD. Circadian times during which BDNF levels in $b d n f^{+/+}$mice were significantly greater $\left({ }^{*} p<0.05\right)$ in comparison with those observed at CT 0,4 , and 8 are indicated. Comparisons of $b d n f^{+/+}$ and $b d n f^{+-}$mice revealed significant differences $(\approx p<0.05)$ in SCN content of BDNF at all times.

the midsubjective day had no phase-resetting action on the rat activity rhythm, indicating that this neurotrophin did not induce the observed phase advances at this circadian time by unmasking the influence of nonphotic inputs to the SCN. Comparison of SCN profiles of BDNF content in control and experimental animals may have some implications for the effect of BDNF inf usion on light-induced phase shifts during the subjective day. In particular, the infusion paradigm does not appear to deliver pharmacological doses of BDNF to the SCN but instead maintains levels consistently within the range of those found in control animals during the subjective night. This infusion-related increase in BDNF levels during the subjective day may effectively gate photic input to the SCN and its phase-shifting action on circadian rhythms by achieving a critical threshold that normally is attained only during the subjective night via endogenous rhythmic amplification of BDNF-mediated signaling. Despite its effects on photic regulation of the pacemaker during the subjective day, BDNF infusion did not potentiate the phase-shifting effect of light during the subjective night perhaps because TrkB receptors in the SCN region are already saturated by the peak expression of endogenous ligand that occurs over this interval (Liang et al., 1998a). Alternatively, this treatment, combined with SCN expression of BDNF, may downregulate TrkB receptor expression in a manner similar to that observed within the midbrain and olfactory bulb (Venero et al., 1994; Frank et al., 1997), thereby obstructing further augmentation of light-induced phase delays or advances during the subjective night.

The converse analysis using BDNF-deficient mutant mice yielded supportive observations on the functional relation between this neurotrophin and photic regulation of the SCN pacemaker. In $b d n f^{+/-}$mice, phase-shifting responses to light during the subjective night were diminished in association with abated BDNF levels and rhythmicity in the SCN. It is noteworthy that this distinction in SCN circadian function of $b d n f^{+/-}$mice was marked by a greater reduction in light-induced phase advances than delays. In accord with previous observations on the relationship between phase response curves to light and circadian period (Daan and Pittendrigh, 1976), this pattern of smaller advances than delays in response to light was correlated with the shorter free-running period of activity rhythm in mutant mice. Although the influence of developmental abnormalities associated with BDNF deficits cannot be excluded, it is important to note that no gross alterations in the histological organization, ganglion cell number, and synaptogenesis of the neural retina or in the structural organization of the CNS have been observed in homozygous BDNF knock-out mice (Jones et al., 1994; Rickman et al., 1998). Moreover, our preliminary analysis has produced accordant observations indicating that basic anatomical and histochemical organization of the SCN are indistinguishable between wild-type and $b d n f^{+/-}$mice. Therefore, it seems unlikely that the decreased phase-shifting responses to light in $b d n f^{+/-}$mice are merely attributable to developmental defects in visual system function or SCN organization.

Involvement of tyrosine kinase receptors in the photic regulation of SCN circadian function is supported by the present finding that light-induced phase shifts of the rat activity rhythm during the subjective night are completely blocked or attenuated by $\mathrm{K} 252 \mathrm{a}$ infusion. TrkB, the cognate receptor for BDNF, is a likely target for K252a action in suppressing phase-shifting responses to light because our anatomical studies demonstrate the localization of TrkB immunoreactivity in the ventral SCN on both fibers emanating from the optic chiasm and cells bordering the chiasm (Liang et al., 1998b). Because this TrkB expression appears to be derived from RHT fibers and SCN cells receiving RHT input, K252a may act to inhibit the peak activity of BDNF during the subjective night on these TrkB receptors and thereby block the endogenous gate for light to induce phase shifts. Importantly, this postulated mechanism of K252a inhibition of photic input and its resetting action on the SCN pacemaker is compatible with recent evidence indicating that this compound blocks BDNF-mediated potentiation of synaptic transmission within other brain regions (Kang and Schuman, 1995; Levine et al., 1995; Akaneya et al., 1997; Carmignoto et al., 1997).

Although these findings establish a role for BDNF in the photic control of circadian rhythms, the mechanism by which this neurotrophin mediates the gating of light input and its phase-shifting effects on the SCN pacemaker is unclear. In the visual cortex and hippocampus, BDNF appears to potentiate neuronal signaling by enhancing transmitter release from presynaptic neurons or the sensitivity of postsynaptic receptors (Levine et al., 1995; Carmignoto et al., 1997; Tanaka et al., 1997). Thus, BDNF regulation of circadian pacemaker responses to light signals may involve similar mechanisms of action on specific neurotransmitter systems that are critical for SCN circadian function. Because glutamate is the primary neurotransmitter of the RHT and mimics the phaseshifting effects of light (Liou et al., 1986; van den Pol, 1993; Shirakawa and Moore, 1994), BDNF could modulate pacemaker responses to photic input via presynaptic enhancement of glutamate release from RHT fibers or postsynaptic modulation of glutamate receptor responsiveness on SCN cells receiving RHT input. This possible action of BDNF in potentiating glutamatergic input to the $\mathrm{SCN}$ is consistent with evidence of BDNF enhancement of AMPA and NMDA receptor-mediated synaptic transmission in hippocampal and visual cortex neurons (Leßmann et al., 1994; Carmignoto et al., 1997). Our reported observations on the close association between BDNF-expressing cells within the ventral SCN and TrkB receptors on fibers emerg- 


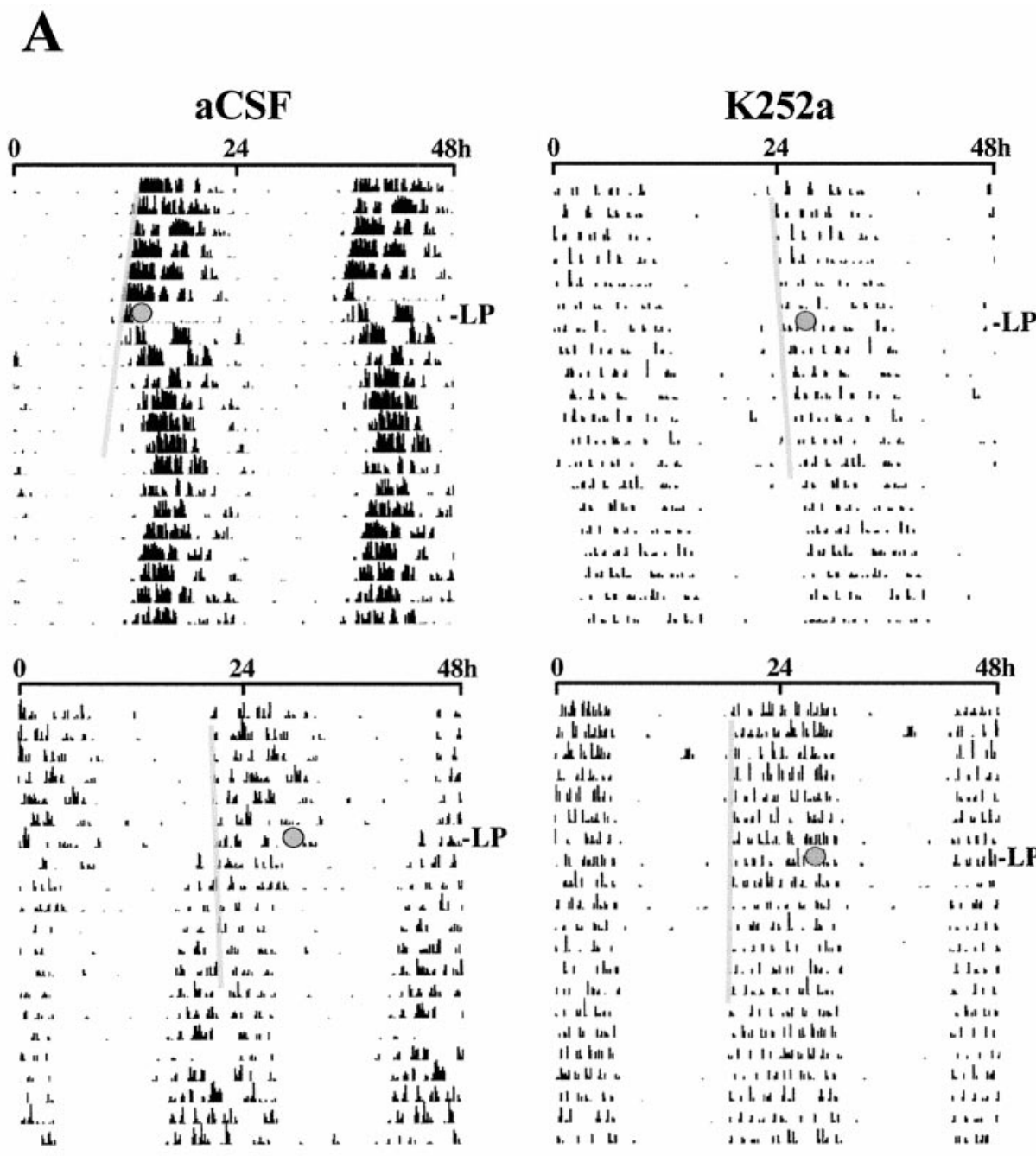

B

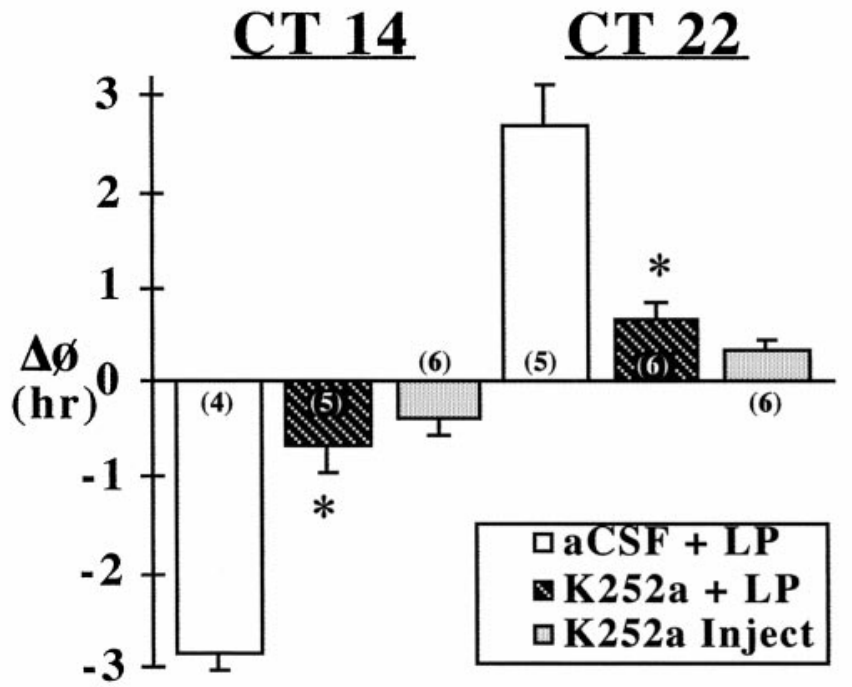

Figure 6. Effects of K252a on light-induced phase shifts of the rat activity rhythm at CT 14 and 22. $A$, Representative activity records of adult male rats receiving infusion of either aCSF or K252a into the SCN for $28 \mathrm{~d}$ during exposure to DD. Actograms are doubleplotted over a $48 \mathrm{hr}$ period, and shaded circles on each record indicate the days and times (top, CT 14; bottom, CT 22) during which animals were exposed to a $30 \mathrm{~min}$ light pulse $(L P)$. A thin shaded bar has been fitted through the activity onsets for 5-6 d before each light pulse to facilitate visualization of the phase shifts in the activity rhythm. $B$, Mean $( \pm$ SEM) phase shift $(\Delta \Phi)$ in hours of the activity rhythm induced by light pulses at CT 14 and 22 in rats receiving SCN infusion of aCSF $(a C S F+L P)$ or K252a $(K 252 a+$ $L P$ ) or by a single injection of K252a (9.34 ng) alone at CT 14 or 22 without exposure to light (K252a Inject). Phase delays are indicated by negative values, and advances are denoted by positive values. For each treatment group, the sample size is designated in parentheses. Light-induced phase delays at CT 14 and advances at CT 22 were significantly decreased $\left({ }^{*} p<0.01\right)$ in K252a-treated rats relative to those in aCSF-infused controls. ing from the optic chiasm and on neighboring cells (Liang et al., 1998 b) indicate that the primary ligand and receptor components for BDNF signal transduction are distributed in the appropriate anatomical relationship. Moreover, BDNF-mediated potentiation of glutamatergic neurotransmission would provide an applicable explanation of its observed effect in enabling light to induce phase advances during the subjective day because glutamate administration into the SCN at this circadian time similarly produces 
comparable advancing shifts of the hamster activity rhythm (Meijer et al., 1988). GABA is another suitable target for the potential action of BDNF in regulating the neurotransmission of photic signals and their effects on the SCN pacemaker because (1) BDNF has been shown to reduce IPSCs markedly and to attenuate $\mathrm{GABA}_{\mathrm{A}}$ receptor-mediated responses to applied GABA in rat hippocampal neurons (Tanaka et al., 1997). (2) Also GABA is the predominant neurotransmitter in the SCN (Moore and Speh, 1993), and GABAergic agents alter the phase-shifting effects of light on circadian rhythms (Ralph and Menaker, 1986, 1989; Golombek and Ralph, 1994). Consequently, BDNF could act via multiple transmitter systems to regulate the circadian resetting of the SCN pacemaker by light.

An alternative possibility is that BDNF could indirectly regulate the phase-shifting effects of light by modulating local cellular interactions or coupling between SCN cells. Throughout the medial SCN, most BDNF-expressing cells are located adjacent to TrkB-immunopositive perikarya (Liang et al., 1998b). Because the SCN contains many cells capable of independent pacemaker function (Welsh et al., 1995), this finding suggests that local paracrine interactions between BDNF and TrkB receptors could provide an endogenous signaling pathway for the intercellular regulation of cellular and metabolic activity within the SCN and thus for pacemaker cell synchronization. Importantly, BDNFmediated changes in pacemaker cell synchronization would be expected to alter $\tau$ and hence modify responses to light. Possible involvement of BDNF in the coupling of SCN pacemaker cells is compatible with the present finding that diminished BDNF expression and rhythmicity in the SCN of heterozygous BDNF knock-out mice are accompanied by decreases in $\tau$ and lightinduced phase shifts relative to that of wild-type mice.

\section{REFERENCES}

Akaneya Y, Tsumoto T, Kinoshita S, Hatanaka H (1997) Brain-derived neurotrophic factor enhances long-term potentiation in rat visual cortex. J Neurosci 17:6707-6716.

Albers HE, Ferris CF (1984) Neuropeptide Y: role in light-dark cycle entrainment of hamster circadian rhythms. Neurosci Lett 50:163-168.

Altar CA, Boylan CB, Jackson C, Hershenson S, Miller J, Wiegand SJ, Lindsay RM, Hyman C (1992) BDNF augments rotational behavior and nigrostriatal dopamine turnover in vivo. Proc Natl Acad Sci USA 89:11347-11351.

Berg MM, Sternberg DW, Parada LF, Chao MV (1992) K-252a inhibits nerve growth factor-induced trk proto-oncogene tyrosine phosphorylation and kinase activity. J Biol Chem 267:13-16.

Bianchi LM, Conover JC, Fritzsch B, DeChiara T, Lindsay RM, Yancopoulos GD (1996) Degeneration of vestibular neurons in late embryogenesis of both heterozygous and homozygous BDNF null mutant mice. Development 122:1965-1973.

Boulos Z, Rusak B (1982) Circadian phase response curves for dark pulses in the hamster. J Comp Physiol [A] 146:411-417.

Carmignoto G, Pizzorusso T, Tia S, Vicini S (1997) BDNF and NGF potentiate excitatory synaptic transmission in the rat visual cortex. J Physiol (Lond) 498:153-164.

Castren E, Zafra F, Thoenen H, Lindholm D (1992) Light regulates expression of brain-derived neurotrophic factor mRNA in rat visual cortex. Proc Natl Acad Sci USA 89:9444-9448.

Chomczynski P (1993) A reagent for the single-step simultaneous isolation of RNA, DNA and proteins from cell and tissue samples. Biotechniques 15:532-534.

Cohen-Cory S, Fraser SE (1995) Effects of brain-derived neurotrophic factor on optic axon branching and remodeling in vivo. Nature 378:192-196.

Conover JC, Erickson JT, Katz DM, Bianchi LM, Poueymirou WT, McClain J, Pan L, Helgren M, Ip NY, Boland P, Friedman B, Wiegand S, Vejsada R, Kato AC, DeChiara TM, Yancopoulos GD (1995) Neuronal deficits, not involving motor neurons, in mice lacking BDNF and/or NT4. Nature 375:235-238.
Croll SD, Wiegand SJ, Anderson KD, Lindsay RM, Nawa H (1994) Regulation of neuropeptides in adult rat forebrain by the neurotrophins BDNF and NGF. Eur J Neurosci 6:1343-1353.

Cui L-N, Dyball REJ (1996) Synaptic input from the retina to the suprachiasmatic nucleus changes with the light-dark cycle in the Syrian hamster. J Physiol (Lond) 497:483-493.

Daan S, Pittendrigh CS (1976) A functional analysis of circadian pacemakers in nocturnal rodents. II. The variability of phase-response curves. J Comp Physiol [A] 106:253-266.

Earnest DJ, Sladek CD (1986) Circadian rhythms of vasopressin release from individual rat suprachiasmatic explants in vitro. Brain Res 382:129-133.

Ellis GB, McKlveen RE, Turek FW (1982) Dark pulses affect the circadian rhythm of activity in hamsters kept in constant light. Am J Physiol 242:R44-R50.

Frank L, Wiegand SJ, Siuciak JA, Lindsay RM, Rudge JS (1997) Effects of BDNF infusion on the regulation of TrkB protein and message in adult rat brain. Exp Neurol 145:62-70.

Golombek DA, Ralph MR (1994) Inhibition of GABA transaminase enhances light-induced circadian phase delays but not advances. J Biol Rhythms 9:251-261.

Jelsma TN, Friedman HH, Berkelaar M, Bray GM, Aguayo AJ (1993) Different forms of the neurotrophin receptor trkB mRNA predominate in rat retina and optic nerve. J Neurobiol 24:1207-1214.

Jones KR, Farinas I, Backus C, Reichardt LF (1994) Targeted disruption of the BDNF gene perturbs brain and sensory neuron development but not motor neuron development. Cell 76:989-999.

Kang H, Schuman EM (1995) Long-lasting neurotrophin-induced enhancement of synaptic transmission in the adult hippocampus. Science 267:1658-1662.

Korte M, Carroll P, Wolf E, Brem G, Thoenen H, Bonhoeffer T (1995) Hippocampal long-term potentiation is impaired in mice lacking brainderived neurotrophic factor. Proc Natl Acad Sci USA 92:8856-8860.

Lehman MN, Jansen HT, Wortman M, Stevens P, Kim C, Norgren RB, Zeitler P (1996) Neurotrophin and neurotrophin receptor expression in the suprachiasmatic nucleus (SCN) of rats and hamsters. Soc Neurosci Abstr 22:1141.

Leßmann V, Gottmann K, Heumann R (1994) BDNF and NT-4/5 enhance glutamatergic synaptic transmission in cultured hippocampal neurones. NeuroReport 6:21-25.

Levine ES, Dreyfus CF, Black IB, Plummer MR (1995) Brain-derived neurotrophic factor rapidly enhances synaptic transmission in hippocampal neurons via postsynaptic tyrosine kinase receptors. Proc Natl Acad Sci USA 92:8074-8077.

Liang F-Q, Walline R, Earnest D (1998a) Circadian rhythm of brainderived neurotrophic factor expression in the rat suprachiasmatic nucleus. Neurosci Lett 242:89-92.

Liang F-Q, Sohrabji F, Miranda R, Earnest B, Earnest D (1998b) Expression of brain-derived neurotrophic factor and its cognate receptor, TrkB, in the rat suprachiasmatic nucleus. Exp Neurol 151:184-193.

Liou SY, Shibata S, Iwasaki K, Ueki S (1986) Optic nerve stimulationinduced increase of release of ${ }^{3} \mathrm{H}$-glutamate and ${ }^{3} \mathrm{H}$-aspartate but not ${ }^{3} \mathrm{H}$-GABA from the suprachiasmatic nucleus in slices of rat hypothalamus. Brain Res Bull 16:427-531.

Meijer JH, van der Zee EA, Dietz M (1988) Glutamate phase shifts circadian activity rhythms in hamsters. Neurosci Lett 86:177-183.

Moore RY (1983) Organization and function of a central nervous system circadian oscillator: the suprachiasmatic nucleus. Fed Proc 42:2783-2789.

Moore RY, Speh JC (1993) GABA is the principal neurotransmitter of the circadian system. Neurosci Lett 150:112-116.

Mrosovsky N (1988) Phase response curves for social entrainment. J Comp Physiol [A] 162:35-46.

Paxinos G, Watson C (1997) The rat brain in stereotaxic coordinates. San Diego: Academic.

Pearson HE, Foley EC, Barbe MF (1995) Localization of Trk receptor immunoreactivity in the visual system of the adult cat. Soc Neurosci Abstr 21:807.

Ralph MR, Menaker M (1986) Effects of diazepam on circadian advances and delays. Brain Res 372:405-408.

Ralph MR, Menaker M (1989) GABA regulation of circadian responses to light. I. Involvement of $\mathrm{GABA}_{\mathrm{A}}$-benzodiazepine and $\mathrm{GABA}_{\mathrm{B}}$ receptors. J Neurosci 9:2858-2865.

Reebs SG, Mrosovsky N (1989) Effects of induced wheel running on the 
circadian activity rhythms of Syrian hamsters: entrainment and phase response curve. J Biol Rhythms 4:39-48.

Rickman DW, Orris A, DeChiara T, Yancopoulos G (1998) Retinal development in the BDNF knockout mouse. Soc Neurosci Abstr 24:295.

Schwartz WJ, Zimmerman P (1990) Circadian timekeeping in BALB/c and C57BL/6 inbred mouse strains. J Neurosci 10:3685-3694.

Shirakawa S, Moore RY (1994) Glutamate shifts the phase of the circadian neuronal firing rhythm in the rat suprachiasmatic nucleus in vitro. Neurosci Lett 178:47-50.

Sofroniew MV, Isacson O, O'Brien TS (1989) Nerve growth factor receptor immunoreactivity in the rat suprachiasmatic nucleus. Brain Res 476:358-362.

Summer TL, Ferraro JS, McCormack CE (1984) Phase-response and Aschoff illuminance curves for locomotor activity of the rat. Am J Physiol 246:R299-R304.

Takahashi JS, DeCoursey PJ, Bauman L, Menaker M (1984) Spectral sensitivity of a novel photoreceptive system mediating entrainment of mammalian circadian rhythms. Nature 308:186-188.

Tanaka T, Saito H, Matsuki N (1997) Inhibition of GABA $A_{A}$ synaptic responses by brain-derived neurotrophic factor in rat hippocampus. J Neurosci 17:2959-2966.

Tapley P, Lamballe E, Barbacid M (1992) K-252a is a selective inhibitor of the tyrosine protein kinase activity of the trk family of oncogenes and neurotrophin receptors. Oncogene 7:371-381.

van den Pol AN (1993) Glutamate and GABA presence and action in the suprachiasmatic nucleus. J Biol Rhythms 8:511-515.

Venero JL, Knusel B, Beck KD, Hefti F (1994) Expression of neurotrophin and trk receptor genes in adult rats with fimbria transections: effect of intraventricular NGF and BDNF administration. Neuroscience 59:797-815.

Welsh DK, Logothetis DE, Meister M, Reppert SM (1995) Individual neurons from rat suprachiasmatic nucleus express independently phased circadian firing rhythms. Neuron 14:697-706. 